\title{
THE DONALDSON-FUTAKI INVARIANT FOR SEQUENCES OF TEST CONFIGURATIONS
}

\author{
TOSHIKI MABUCHI*
}

\begin{abstract}
In this note, given a polarized algebraic manifold $(X, L)$, we define the Donaldson-Futaki invariant $F_{1}\left(\left\{\mu_{i}\right\}\right)$ for a sequence $\left\{\mu_{i}\right\}$ of test configurations for $(X, L)$ of exponents $\ell_{j}$ satisfying

$$
\ell_{j} \rightarrow \infty, \quad \text { as } j \rightarrow \infty \text {. }
$$

This then allows us to define a strong version of K-stability or Ksemistability for $(X, L)$. In particular, $(X, L)$ will be shown to be $\mathrm{K}$ semistable in this strong sense if the polarization class $c_{1}(L)_{\mathbb{R}}$ admits a constant scalar curvature Kähler metric.
\end{abstract}

\section{INTRODUCTION}

By a polarized algebraic manifold $(X, L)$, we mean a pair of a nonsingular irreducible projective variety $X$, defined over $\mathbb{C}$, and a very ample line bundle $L$ over $X$. Replacing $L$ by its suitable multiple if necessary, we may assume

$$
H^{q}\left(X, L^{\otimes \ell}\right)=\{0\}, \quad q=1,2, \ldots, n,
$$

for all positive integers $\ell$, where $n:=\operatorname{dim}_{\mathbb{C}} X$. In this note, we fix once for all such a pair $(X, L)$. For the affine line $\mathbb{A}^{1}:=\{z \in \mathbb{C}\}$, we consider the algebraic torus $T:=\mathbb{C}^{*}$ acting on $\mathbb{A}^{1}$ by multiplication of complex numbers,

$$
T \times \mathbb{A}^{1} \rightarrow \mathbb{A}^{1}, \quad(t, z) \mapsto t z .
$$

For each positive integer $\ell$, the space $V_{\ell}:=H^{0}\left(X, L^{\otimes \ell}\right)$ is assumed to have a Hermitian metric $\rho_{\ell}$ expressible in the form (1.6) below. Let

$$
\Phi_{\ell}: X \hookrightarrow \mathbb{P}^{*}\left(V_{\ell}\right)
$$

be the Kodaira embedding of $X$ associated to the complete linear system $\left|L^{\otimes \ell}\right|$ on $X$. By fixing an $\ell$, we consider an algebraic group homomorphism

$$
\psi: T \rightarrow \mathrm{GL}\left(V_{\ell}\right)
$$

*Supported by JSPS Grant-in-Aid for Scientific Research (A) No. 20244005. 
such that the maximal compact subgroup $S^{1} \subset \mathbb{C}^{*}(=T)$ acts isometrically on $\left(V_{\ell}, \rho_{\ell}\right)$. Let $\mathcal{X}^{\psi}$ be the irreducible algebraic subvariety of $\mathbb{A}^{1} \times \mathbb{P}^{*}\left(V_{\ell}\right)$ obtained as the closure of $\cup_{z \in T} \mathcal{X}_{z}^{\psi}$ in $\mathbb{A}^{1} \times \mathbb{P}^{*}\left(V_{\ell}\right)$ by setting

$$
\mathcal{X}_{z}^{\psi}:=\{z\} \times \psi(z) \Phi_{\ell}(X), \quad z \in T
$$

where the element $\psi(z)$ in $\operatorname{GL}\left(V_{\ell}\right)$ acts naturally on the set $\mathbb{P}^{*}\left(V_{\ell}\right)$ of all hyperplanes in $V_{\ell}$ passing through the origin. We then consider the map

$$
\pi: \mathcal{X}^{\psi} \rightarrow \mathbb{A}^{1}
$$

induced by the projection of $\mathbb{A}^{1} \times \mathbb{P}^{*}\left(V_{\ell}\right)$ to the first factor $\mathbb{A}^{1}$. Moreover, for the hyperplane bundle $\mathcal{O}_{\mathbb{P}^{*}\left(V_{\ell}\right)}(1)$ on $\mathbb{P}^{*}\left(V_{\ell}\right)$, we put

$$
\mathcal{L}^{\psi}:=\operatorname{pr}_{2}^{*} \mathcal{O}_{\mathbb{P}^{*}\left(V_{\ell}\right)}(1)_{\mid \mathcal{X}^{\psi}},
$$

where $\operatorname{pr}_{2}: \mathbb{A}^{1} \times \mathbb{P}^{*}\left(V_{\ell}\right) \rightarrow \mathbb{P}^{*}\left(V_{\ell}\right)$ is the projection to the second factor. For the dual vector space $V_{\ell}^{*}$ of $V_{\ell}$, the $\mathbb{C}^{*}$-action on $\mathbb{A}^{1} \times V_{\ell}^{*}$ defined by

$$
\mathbb{C}^{*} \times\left(\mathbb{A}^{1} \times V_{\ell}^{*}\right) \rightarrow \mathbb{A}^{1} \times V_{\ell}^{*}, \quad(t,(z, p)) \mapsto(t z, \psi(t) p),
$$

naturally induces a $\mathbb{C}^{*}$-action on $\mathbb{A}^{1} \times \mathbb{P}^{*}\left(V_{\ell}\right)$ and $\mathcal{O}_{\mathbb{P}^{*}\left(V_{\ell}\right)}(-1)$, where $\operatorname{GL}\left(V_{\ell}\right)$ acts on $V_{\ell}^{*}$ by contragradient representation. This then induces $\mathbb{C}^{*}$-actions on $\mathcal{X}^{\psi}$ and $\mathcal{L}^{\psi}$, and $\pi: \mathcal{X}^{\psi} \rightarrow \mathbb{A}^{1}$ is a projective morphism with relative very ample line bundle $\mathcal{L}^{\psi}$ such that

$$
\left(\mathcal{X}_{z}^{\psi}, \mathcal{L}_{z}^{\psi}\right) \cong\left(X, L^{\otimes \ell}\right), \quad z \neq 0
$$

where for each $z \in \mathbb{A}^{1}$, we denote by $\mathcal{L}_{z}^{\psi}$ the restriction of $\mathcal{L}^{\psi}$ to the schemetheoretic fiber $\mathcal{X}_{z}^{\psi}$ of $\mathcal{X}^{\psi}$ over $z$. Then a triple $(\mathcal{X}, \mathcal{L}, \psi)$ is called a test configuration for $(X, L)$, if we have both

$$
\mathcal{X}=\mathcal{X}^{\psi} \quad \text { and } \quad \mathcal{L}=\mathcal{L}^{\psi}
$$

for some $\psi$ as above, where $\ell$ is called the exponent of the test configuration $(\mathcal{X}, \mathcal{L}, \psi)$. We consider the projective linear representation

$$
\psi^{\mathrm{PGL}}: T \rightarrow \mathrm{PGL}\left(V_{\ell}\right)
$$

induced by $\psi$. Then a test configuration $(\mathcal{X}, \mathcal{L}, \psi)$ for $(X, L)$ is called trivial, if $\psi^{\mathrm{PGL}}$ is a trivial homomorphism. We now consider the set $\mathcal{M}$ of all sequences $\left\{\mu_{j}\right\}$ of test configurations

$$
\mu_{j}=\left(\mathcal{X}_{j}, \mathcal{L}_{j}, \psi_{j}\right), \quad j=1,2, \ldots
$$


for $(X, L)$ such that the exponent $\ell_{j}$ of the test configuration $\mu_{j}$ satisfies the following growth condition:

$$
\ell_{j} \rightarrow+\infty, \text { as } j \rightarrow \infty
$$

Assume now that $\ell_{1}=1$ for simplicity. In Section 2 , for every element $\left\{\mu_{j}\right\}$ in $\mathcal{M}$, we shall define the Donaldson-Futaki invariant

$$
F_{1}\left(\left\{\mu_{j}\right\}\right) \in \mathbb{R} \cup\{-\infty\} .
$$

This definition is justified in the sense that $F_{1}\left(\left\{\mu_{j}\right\}\right) \in \mathbb{R} \cup\{-\infty\}$ can be viewed as the Donaldson-Futaki invariant $F_{1}\left(\mu_{\infty}\right)$ for the limit $\mu_{\infty}$ of a suitable subsequence of $\left\{\mu_{j}\right\}$ in some completion of the moduli space of test configurations for $(X, L)$. This will be shown in [7] in general, while the most important case is the following:

For a $T$-action on an irreducible algebraic variety $\mathcal{X}$, we assume that there exists a $T$-equivariant projective morphism

$$
\pi: \mathcal{X} \rightarrow \mathbb{A}^{1}
$$

with a relatively very ample line bundle $\mathcal{L}$ on the fiber space $\mathcal{X}$ over $\mathbb{A}^{1}$ such that the $T$-action on $\mathcal{X}$ lifts to a $T$-linearization of $\mathcal{L}$. Let $(\mathcal{X}, \mathcal{L})$ be a test configuration for $(X, L)$ of exponent 1 in Donaldson's sense (see [1]), i.e., we have an algebraic isomorphism

$$
\left(\mathcal{X}_{z}, \mathcal{L}_{z}\right) \cong(X, L), \quad 0 \neq z \in \mathbb{A}^{1}
$$

where $\mathcal{L}_{z}$ denotes the restriction of $\mathcal{L}$ to $\mathcal{X}_{z}:=\pi^{-1}(z)$. Then by the affirmative solution of equivariant Serre's conjecture, the direct image sheaves

$$
E^{j}:=\pi_{*} \mathcal{L}^{\otimes \ell_{j}}, \quad j=1,2, \ldots,
$$

can be viewed as the trivial vector bundles over $\mathbb{A}^{1}$ in terms of a $T$-equivariant isomorphism of vector bundles

$$
E^{j} \cong \mathbb{A}^{1} \times E_{0}^{j}
$$

where the Hermitian metric $\rho_{\ell_{j}}$ on the fiber $E_{1}^{j}\left(=V_{\ell_{j}}\right)$ over 1 is chosen to be $\rho_{h}^{\left(\ell_{j}\right)}$ in (1.6) inducing a Hermitian metric on the central fiber $E_{0}^{\ell}$ which is preserved by the action of $S^{1} \subset T$ on $E_{0}^{j}$ (see [2]). Here the $T$-action on $\mathbb{A}^{1} \times E_{0}^{j}$ is induced by the $T$-action (1.1) on $\mathbb{A}^{1}$ and a natural $T$-representation

$$
\psi_{j}: T \rightarrow \underset{3}{\operatorname{GL}\left(V_{j}\right)}\left(=\mathrm{GL}\left(E_{0}^{j}\right)\right),
$$


under the identification of $E_{0}^{j}$ with $E_{1}^{j}\left(=V_{j}\right)$ by the isomorphism (1.4). In view of $\ell_{1}=1$, we write $\psi_{1}$ simply as $\psi$. We now assume that the test configuration $(\mathcal{X}, \mathcal{L})$ is nontrivial, i.e., the homomorphism $\psi^{\mathrm{PGL}}$ is nontrivial. Put $\lambda=c_{1}(X)^{n}[X] / \pi$. The following will be proved in a separate paper:

Fact (cf. [6]). For the test configuration $(\mathcal{X}, \mathcal{L})$ as above for $(X, L)$ in Donaldson's sense, consider the sequence of test configurations $\mu_{j}=\left(\mathcal{X}_{j}, \mathcal{L}_{j}, \psi_{j}\right)$ of exponents $\ell_{j}, j=1,2, \ldots$, defined by

$$
\mathcal{X}_{j}:=\mathcal{X} \quad \text { and } \quad \mathcal{L}_{j}:=\mathcal{L}^{\otimes \ell_{j}},
$$

where $\ell_{j}, j=1,2, \ldots$, are positive integers satisfying $\ell_{1}=1$ and (1.3). Replacing the sequence $\left\{\ell_{j}\right\}$ by its suitable subsequence if necessary, in view of $\left[5 ;\right.$ Remark 4.6] and [6; Lemma 2.6], we may assume that $\left|b_{j}\right|_{1}$ in (2.2) converges to a nonnegative real number $\beta$. If $\beta>0$, then for the sequence $\left\{\mu_{j}\right\}$ of test configurations, we have

$$
F_{1}\left(\left\{\mu_{j}\right\}\right)=\beta^{-1} \lambda F_{1}(\mathcal{X}, \mathcal{L}),
$$

where $F_{1}(\mathcal{X}, \mathcal{L})$ on the right-hand side is the Donaldson-Futaki invariant of the test configuration $(\mathcal{X}, \mathcal{L})$ in Donaldson's sense.

Now we come back to a general situation. Fix a Hermitian metric $h$ for $L$ such that $\omega:=c_{1}(L ; h)$ is Kähler. For each positive integer $\ell$, we choose $\rho_{\ell}$ as the Hermitian metric $\rho_{h}^{(\ell)}$ on $V_{\ell}$ defined by

$$
\rho_{h}^{(\ell)}\left(\sigma^{\prime}, \sigma^{\prime \prime}\right):=\int_{X}\left(\sigma^{\prime}, \sigma^{\prime \prime}\right)_{h} \omega^{n}, \quad \sigma^{\prime}, \sigma^{\prime \prime} \in V_{\ell},
$$

where $\left(\sigma^{\prime}, \sigma^{\prime \prime}\right)_{h}$ denotes the pointwise Hermitian inner product of $\sigma$ and $\sigma^{\prime}$ by the $\ell$-multiple of $h$. We shall now define a strong version of K-stability which will be studied in detail in a forthcoming paper [5].

Definition 1.7. (1) A polarized algebraic manifold $(X, L)$ is called strongly K-semistable, if $F_{1}\left(\left\{\mu_{j}\right\}\right) \leq 0$ for all $\left\{\mu_{j}\right\} \in \mathcal{M}$.

(2) A strongly K-semistable polarized algebraic manifold $(X, L)$ is called strongly $K$-stable, if for every $\left\{\mu_{j}\right\} \in \mathcal{M}$ satisfying $F_{1}\left(\left\{\mu_{j}\right\}\right)=0$, there exists a $j_{0}$ such that the test configurations $\mu_{j}$ are trivial for all $j \geq j_{0}$.

We here observe that these definitions of strong K-semistability and strong K-stability are independent of the choice of $h$ above (cf. [7]). Finally in Section 4, we shall prove the following:

Theorem. If the polarization class $c_{1}(L)_{\mathbb{R}}$ admits a constant scalar curvature Kähler metric, then $(X, L)$ is strongly K-semistable. 
In [5], we shall show a stronger result that the polarized algebraic manifold $(X, L)$ in the Theorem above is actually strongly $\mathrm{K}$-stable.

\section{The Donaldson-Futaki invariant $F_{1}$ On $\mathcal{M}$}

Let $\mu_{j}=\left(\mathcal{X}_{j}, \mathcal{L}_{j}, \psi_{j}\right), j=1,2, \ldots$, be a sequence of test configurations for $(X, L)$ as in (1.2), and let $\ell_{j}$ be the exponent of $\mu_{j}$. By abuse of terminology, we write the vector space $V_{\ell_{j}}=H^{0}\left(X, L^{\otimes \ell_{j}}\right) \operatorname{simply}$ as $V_{j}$. Put $N_{j}:=\operatorname{dim} V_{j}$. Then by setting $d_{j}:=\ell_{j}^{n} c_{1}(L)^{n}[X]$, we define

$$
W_{j}:=\left\{\operatorname{Sym}^{d_{j}}\left(V_{j}\right)\right\}^{\otimes n+1},
$$

where $\operatorname{Sym}^{d_{j}}\left(V_{j}\right)$ denotes the $d_{j}$-th symmetric tensor product of $V_{j}$. The dual space $W_{j}^{*}$ of $W_{j}$ admits the Chow norm (cf. [12])

$$
W_{j}^{*} \ni w \mapsto\|w\|_{\mathrm{CH}\left(\rho_{\ell_{j}}\right)} \in \mathbb{R}_{\geq 0},
$$

associated to the Hermitian metric $\rho_{\ell_{j}}$ on $V_{j}$ as in (1.6). For the Kodaira embedding $\Phi_{j}: X \hookrightarrow \mathbb{P}^{*}\left(V_{j}\right)$ associated to the complete linear system $\left|L^{\otimes \ell_{j}}\right|$ on $X$, we consider the Chow form

$$
0 \neq \hat{X}_{j} \in W_{j}^{*}
$$

for the irreducible reduced algebraic cycle $\Phi_{j}(X)$ on $\mathbb{P}^{*}\left(V_{j}\right)$ such that the corresponding point $\left[\hat{X}_{j}\right]$ in $\mathbb{P}^{*}\left(W_{j}\right)$ is the Chow point for the cycle $\Phi_{j}(X)$. For the homomorphism $\psi_{j}: T \rightarrow \mathrm{GL}\left(V_{j}\right)$, taking the real Lie subgroup

$$
T_{\mathbb{R}}=\left\{t \in T ; t \in \mathbb{R}_{+}\right\}
$$

of the algebraic torus $T=\left\{t \in \mathbb{C}^{*}\right\}$, we now define a Lie group homomorphism $\psi_{j}^{\mathrm{SL}}: T_{\mathbb{R}} \rightarrow \mathrm{SL}\left(V_{j}\right)$ by

$$
\psi_{j}^{\mathrm{SL}}(t):=\frac{\psi_{j}(t)}{\operatorname{det}\left(\psi_{j}(t)\right)^{1 / N_{j}}}, \quad t \in T_{\mathbb{R}} .
$$

Then for a suitable orthonormal basis $\left\{\sigma_{\alpha} ; \alpha=1,2, \ldots, N_{j}\right\}$ for $V_{j}$, there exist rational numbers $b_{j, \alpha}, \alpha=1,2, \ldots, N_{j}$ such that the homomorphism $\psi_{j}^{\mathrm{SL}}: T_{\mathbb{R}} \rightarrow \mathrm{SL}\left(V_{j}\right)$ is written in the form

$$
\psi_{j}^{\mathrm{SL}}(t) \cdot \sigma_{\alpha}=t^{-b_{j, \alpha}} \sigma_{\alpha}, \quad \alpha=1,2, \ldots, N_{j},
$$

where $t \in \mathbb{R}_{+}$is arbitrary. Then for $b_{j}:=\left(b_{j, 1}, b_{j, 2}, \ldots, b_{j, N_{j}}\right) \in \mathbb{R}^{N_{j}}$ as above, we shall now define its norms

$$
\left\{\begin{array}{c}
\left|b_{j}\right|_{1}:=\Sigma_{\alpha=1}^{N_{j}}\left|b_{j, \alpha}\right| / \ell_{j}^{n+1} \\
\left|b_{j}\right|_{\infty}:=\max \left\{\left|b_{j, 1}\right|,\left|b_{j, 2}\right|, \ldots, \mid b_{\left.j, N_{j} \mid\right\} / \ell_{j}}\right.
\end{array}\right.
$$


where by the definition of $b_{j}$ we have the equality

$$
b_{j, 1}+b_{j, 2}+\cdots+b_{j, N_{j}}=0 .
$$

Since $\operatorname{SL}\left(V_{j}\right)$ acts naturally on $W_{j}^{*}$, writing each $t \in T_{\mathbb{R}}$ as $t=\exp \left(s /\left|b_{j}\right|_{\infty}\right)$ for some $s \in \mathbb{R}$ in the case $\left|b_{j}\right|_{\infty} \neq 0$, we define a real function $f_{j}$ on $\mathbb{R}$ by

$$
f_{j}(s):= \begin{cases}\left(\left|b_{j}\right|_{1} \ell_{j}^{n}\right)^{-1}\left|b_{j}\right|_{\infty} \log \left\|\psi_{j}^{\mathrm{SL}}(t) \cdot \hat{X}_{j}\right\|_{\mathrm{CH}\left(\rho_{\ell_{j}}\right)}, & \text { if } \psi_{j}^{\mathrm{PGL}} \neq 1, \\ \ell_{j}^{-n} \log \left\|\hat{X}_{j}\right\|_{\mathrm{CH}\left(\rho_{\ell_{j}}\right)}, & \text { if } \psi_{j}^{\mathrm{PGL}}=1,\end{cases}
$$

where $s$ runs through the set of all real numbers. Put $\dot{f}_{j}:=d f_{j} / d s$. Since $h$ is fixed, the derivative $\dot{f}_{j}(0)$ at $s=0$ is bounded from above by a constant $C>0$ independent of $j$ (see Section 3). Define $F_{1}\left(\left\{\mu_{j}\right\}\right) \in \mathbb{R} \cup\{-\infty\}$ by

$$
F_{1}\left(\left\{\mu_{j}\right\}\right):=\lim _{s \rightarrow-\infty}\left\{\varliminf_{j \rightarrow \infty} \dot{f}_{j}(s)\right\} \leq C .
$$

This is well-defined, because the function $\underline{\lim }_{j \rightarrow \infty} \dot{f}_{j}(s)$ is non-decreasing in $s$ by convexity of the functions $f_{j}$ (cf. [12]; see also [4], Theorem 4.5).

\section{BOUNDEDNESS FROM ABOVE FOR $\dot{f}_{j}(0)$}

If $\psi_{j}^{\mathrm{PGL}}=1$, then $f_{j}$ is a constant function, so that $\dot{f}_{j}(0)=0$. Hence we may assume that $\psi_{j}^{\mathrm{PGL}} \neq 1$. For the Kähler metric $\omega=c_{1}(L ; h)$ on $X$, let $B_{j}(\omega)$ denote the $\ell_{j}$-th asymptotic Bergman kernel defined by

$$
B_{j}(\omega):=\Sigma_{\alpha=1}^{N_{j}}\left|\sigma_{\alpha}\right|_{h}^{2}
$$

for the orthonormal basis $\left\{\sigma_{\alpha} ; \alpha=1,2, \ldots, N_{j}\right\}$ of $V_{j}$ as in Section 2, where we put $\left|\sigma_{\alpha}\right|_{h}^{2}:=\left(\sigma_{\alpha}, \sigma_{\alpha}\right)_{h}$ for simplicity. We now consider the Kodaira embedding $\Phi_{j}: X \hookrightarrow \mathbb{P}^{N_{j}-1}(\mathbb{C})=\left\{\left(z_{1}: z_{2}: \cdots: z_{N_{j}}\right)\right\}$ of $X$ in the projective space $\mathbb{P}^{N_{j}-1}(\mathbb{C})$ defined by

$$
\Phi_{j}(x):=\left(\sigma_{1}(x): \sigma_{2}(x): \cdots: \sigma_{N_{j}}(x)\right), \quad x \in X,
$$

where we identify $\mathbb{P}^{*}\left(V_{j}\right)=\mathbb{P}^{N_{j}-1}(\mathbb{C})$. Let $\omega_{\mathrm{FS}}:=(\sqrt{-1} / 2 \pi) \partial \bar{\partial} \log \Sigma_{\alpha=1}^{N_{j}}\left|z_{\alpha}\right|^{2}$ be the Fubini-Study form on $\mathbb{P}^{N_{j}-1}(\mathbb{C})$. Then by [1], we see that

$$
\begin{aligned}
& \left|B_{j}(\omega)-(1 / n !) \ell_{j}^{n}\right| \leq C_{1} \ell_{j}^{n-1}, \\
& \left(\ell_{j}-C_{2}\right) \omega \leq \Phi_{j}^{*} \omega_{\mathrm{FS}} \leq\left(\ell_{j}+C_{2}\right) \omega,
\end{aligned}
$$

where $C_{i}, i=1,2, \ldots$, denote positive real constants independent of the choice of $j, \alpha$ and $t \in \mathbb{C}^{*}$ throughout this paper. By [12] (see also [4]),

$$
\dot{f}_{j}(0)=\left(\left|b_{j}\right|_{1} \ell_{j}^{n}\right)^{-1} \int_{X} B_{j}(\omega)^{-1}\left(\Sigma_{\alpha=1}^{N_{j}} b_{j, \alpha}\left|\sigma_{\alpha}\right|_{h}^{2}\right) \Phi_{j}^{*} \omega_{\mathrm{FS}}^{n},
$$


where the norm $\left|b_{j}\right|_{1}$ is as in (2.2). Then $\left(\left|b_{j}\right|_{1} \ell_{j}\right)^{-1} \int_{X} \Sigma_{\alpha=1}^{N_{j}} b_{j, \alpha}\left|\sigma_{\alpha}\right|_{h}^{2} \omega^{n}=0$ by (2.3). Hence by setting

$$
\left\{\begin{array}{l}
R_{1}:=\left(\left|b_{j}\right|_{1} \ell_{j}^{n}\right)^{-1} \int_{X} B_{j}(\omega)^{-1}\left(\Sigma_{\alpha=1}^{N_{j}} b_{j, \alpha}\left|\sigma_{\alpha}\right|_{h}^{2}\right)\left\{\Phi_{j}^{*} \omega_{\mathrm{FS}}^{n}-\left(\ell_{j} \omega\right)^{n}\right\}, \\
R_{2}:=\left(\left|b_{j}\right|_{1} \ell_{j}^{n}\right)^{-1} \int_{X}\left\{B_{j}(\omega)^{-1}-\left(\ell_{j}^{n} / n !\right)^{-1}\right\}\left(\Sigma_{\alpha=1}^{N_{j}} b_{j, \alpha}\left|\sigma_{\alpha}\right|_{h}^{2}\right)\left(\ell_{j} \omega\right)^{n}
\end{array}\right.
$$

we see from (3.4) that $\dot{f}_{j}(0)=R_{1}+R_{2}$. Now by (3.2) and (3.3),

$$
\begin{aligned}
R_{1} & \leq \ell_{j}\left(\Sigma_{\alpha=1}^{N_{j}}\left|b_{j, \alpha}\right|\right)^{-1} \int_{X} B_{j}(\omega)^{-1}\left(\Sigma_{\alpha=1}^{N_{j}}\left|b_{j, \alpha}\right|\left|\sigma_{\alpha}\right|_{h}^{2}\right)\left|\Phi_{j}^{*} \omega_{\mathrm{FS}}^{n}-\left(\ell_{j} \omega\right)^{n}\right| \\
& \leq C_{3} \ell_{j}\left(\Sigma_{\alpha=1}^{N_{j}}\left|b_{j, \alpha}\right|\right)^{-1} \int_{X}\left(\ell_{j}^{n} / n !\right)^{-1}\left(\Sigma_{\alpha=1}^{N_{j}}\left|b_{j, \alpha}\right|\left|\sigma_{\alpha}\right|_{h}^{2}\right) \ell_{j}^{n-1} \omega^{n} \\
& =n ! C_{3}\left(\Sigma_{\alpha=1}^{N_{j}}\left|b_{j, \alpha}\right|\right)^{-1} \int_{X}\left(\Sigma_{\alpha=1}^{N_{j}}\left|b_{j, \alpha}\right|\left|\sigma_{\alpha}\right|_{h}^{2}\right) \omega^{n}=n ! C_{3} .
\end{aligned}
$$

On the other hand, we see from (3.2) that

$$
\begin{aligned}
R_{2} & \leq C_{4} \ell_{j}\left(\Sigma_{\alpha=1}^{N_{j}}\left|b_{j, \alpha}\right|\right)^{-1} \int_{X} \ell_{j}^{-n-1}\left(\Sigma_{\alpha=1}^{N_{j}} b_{j, \alpha}\left|\sigma_{\alpha}\right|_{h}^{2}\right)\left(\ell_{j} \omega\right)^{n} \\
& \leq C_{4}\left(\Sigma_{\alpha=1}^{N_{j}}\left|b_{j, \alpha}\right|\right)^{-1} \int_{X}\left(\Sigma_{\alpha=1}^{N_{j}}\left|b_{j, \alpha}\right|\left|\sigma_{\alpha}\right|_{h}^{2}\right) \omega^{n}=C_{4} .
\end{aligned}
$$

Thus we obtain $\dot{f}_{j}(0)=R_{1}+R_{2} \leq n ! C_{3}+C_{4}=C_{5}$, as required.

\section{Proof of Theorem}

In this section, we use the same notation as in Section 3 by choosing a Kähler metric of constant scalar curvature as our $\omega=c_{1}(L ; h)$. Associated to this $h$, we take $\rho_{h}^{(\ell)}$ in (1.6) as the metric $\rho_{\ell}$ on $V_{\ell}$. The scalar curvature

$$
S_{\omega}=n c_{1}(M) c_{1}(L)^{n-1}[X] / c_{1}(L)^{n}[X]
$$

of $\omega$ is a constant function independent of the choice of $j$. Now by [3] and [11, the $\ell_{j}$-th asymptotic Bergman kernel $B_{j}(\omega)$ in (3.1) is written as

$$
B_{j}(\omega)=(1 / n !)\left\{\ell_{j}^{n}+\left(S_{\omega} / 2\right) \ell_{j}^{n-1}+R_{j} \ell_{j}^{n-2}\right\}
$$

where $R_{j}$ is a function on $X$ satisfying $\left\|R_{j}\right\|_{C^{2}(X)} \leq C_{6}$. Now for each integer $r$, let $O\left(\ell_{j}^{r}\right)$ denote a function $\varphi$ or a real 2-form $\theta$ on $X$ such that $|\varphi| \leq C_{7} \ell_{j}^{r}$ or $-C_{7} \ell_{j}^{r} \omega \leq \theta \leq C_{7} \ell_{j}^{r} \omega$, respectively, for some $C_{7}$. Then by taking $(\sqrt{-1} / 2 \pi) \partial \bar{\partial} \log$ of both sides of (4.1), we obtain

$$
\Phi_{j}^{*} \omega_{\mathrm{FS}}-\ell_{j} \omega=O\left(\ell_{j}^{-2}\right)
$$


Hence by (4.1) and (4.2), we see from (3.4) that

$$
\begin{aligned}
\dot{f}_{j}(0) & =\left(\left|b_{j}\right|_{1} \ell_{j}^{n}\right)^{-1} \int_{X} \frac{n ! \Sigma_{\alpha=1}^{N_{j}} b_{j, \alpha}\left|\sigma_{\alpha}\right|_{h}^{2}}{\ell_{j}^{n}+\left(S_{\omega} / 2\right) \ell_{j}^{n-1}+O\left(\ell_{j}^{n-2}\right)}\left\{\ell_{j} \omega+O\left(\ell_{j}^{-2}\right)\right\}^{n} \\
& =\frac{n !\left(\left|b_{j}\right|_{1} \ell_{j}^{n}\right)^{-1}}{\ell_{j}^{n}+\left(S_{\omega} / 2\right) \ell_{j}^{n-1}} \int_{X} \frac{\Sigma_{\alpha=1}^{N_{j}} b_{j, \alpha}\left|\sigma_{\alpha}\right|_{h}^{2}}{1+O\left(\ell_{j}^{-2}\right)}\left\{\ell_{j} \omega+O\left(\ell_{j}^{-2}\right)\right\}^{n} .
\end{aligned}
$$

Since $\int_{X} \Sigma_{\alpha=1}^{N_{j}} b_{j, \alpha}\left|\sigma_{\alpha}\right|_{h}^{2} \omega^{n}=\Sigma_{\alpha=1}^{N_{j}} b_{j, \alpha}=0$ by (2.3), it now follows that

$$
\begin{aligned}
\left|\dot{f}_{j}(0)\right| & \leq C_{8}\left(\left|b_{j}\right|_{1} \ell_{j}^{n}\right)^{-1} \int_{X}\left(\Sigma_{\alpha=1}^{N_{j}}\left|b_{j, \alpha}\right|\left|\sigma_{\alpha}\right|_{h}^{2}\right) O\left(\ell_{j}^{-2}\right) \omega^{n} \\
& =C_{8}\left(\left|b_{j}\right|_{1} \ell_{j}^{n}\right)^{-1}\left(\Sigma_{\alpha=1}^{N_{j}}\left|b_{j, \alpha}\right|\right) O\left(\ell_{j}^{-2}\right)=O\left(\ell_{j}^{-1}\right),
\end{aligned}
$$

where the last equality follows from (2.2). Then by $\lim _{j \rightarrow \infty} \ell_{j}^{-1}=0$, we obtain $\lim _{j \rightarrow \infty} \dot{f}_{j}(0)=0$. Since $\underline{\lim }_{j \rightarrow \infty} \dot{f}_{j}(s)$ is a non-decreasing function in $s$, by (2.4), we now conclude for all $\left\{\mu_{j}\right\} \in \mathcal{M}$ that

$$
F_{1}\left(\left\{\mu_{j}\right\}\right)=\lim _{s \rightarrow-\infty}\left\{\varliminf_{j \rightarrow \infty} \dot{f}_{j}(s)\right\} \leq \varliminf_{j \rightarrow \infty} \dot{f}_{j}(0)=0,
$$

as required. This completes the proof of Theorem.

\section{REFERENCES}

[1] S.K. Donaldson: Scalar curvature and stability of toric varieties, J. Differential Geom. 62 (2002), 289-349.

[2] S.K. Donaldson: Lower bounds on the Calabi functional, J. Differential Geom. 70 (2005), 453-472.

[3] Z. Lu: On the lower order terms of the asymptotic expansion of Tian-Yau-Zelditch, Amer. J. Math. 122 (2000), 235-273.

[4] T. Mabuchi: Stability of extremal Kähler manifolds, Osaka J. Math. 41 (2004), 563-582.

[5] T. Mabuchi: A stronger concept of K-stability, a revised version of arXiv: 0910.4617, in preparation.

[6] T. Mabuchi: A remark on the Donaldson-Futaki invariant for sequences of test configurations, in preparation.

[7] T. Mabuchi And Y. NitTa: Completion of the moduli space of test configurations, in preparation.

[8] T. Mabuchi And Y. NitTa: Strong K-stability and asymptotic Chow-stability, arXiv: 1307.1959 .

[9] Y. SAno: On stability criterion of complete intersections J. Geom. Anal. 14 (2004), 533-544.

[10] G. SzÉKelyhidi: Filtrations and test-configurations, arXiv:1111.4986v2.

[11] S. Zelditch: Szegö kernels and a theorem of Tian, Int. Math. Res. Not. 6 (1998), $317-331$. 
[12] S. Zhang: Heights and reductions of semi-stable varieties, Compositio Math. 104 (1996), 77-105.

Department of Mathematics

OSAKA UNIVERSITY

TOYONAKA, OSAKA, 560-0043

JAPAN 